\title{
Linx
}

Revue des linguistes de l'université Paris X Nanterre

9 | 1997

Émile Benveniste. Vingt ans après

\section{Benveniste et Kurylowicz : deux méthodes, deux trouvailles sur le système aspecto-temporel}

Annie Montaut

\section{(2) OpenEdition}

\section{Journals}

Édition électronique

URL : http://journals.openedition.org/linx/1080

DOI : 10.4000/linx.1080

ISSN : 2118-9692

Éditeur

Presses universitaires de Paris Nanterre

\section{Édition imprimée}

Date de publication : 1 avril 1997

Pagination : 337-353

ISSN : 0246-8743

\section{Référence électronique}

Annie Montaut, « Benveniste et Kurylowicz : deux méthodes, deux trouvailles sur le système aspectotemporel », Linx [En ligne], 9 | 1997, mis en ligne le 09 juillet 2012, consulté le 21 avril 2019. URL http://journals.openedition.org/linx/1080 ; DOI : 10.4000/linx.1080 


\title{
Benveniste et Kurylowicz : deux méthodes, deux trouvailles sur le système aspecto-temporel
}

\author{
Annie Montaut
}

$\mathrm{L}$

a même année - 1965 - Benveniste et Kurylowicz écrivent dans la même revue - Diogène - deux articles, en français, portant sur le même problème, à savoir le temps linguistique, où il est beaucoup question du rapport du parfait et du futur au présent ${ }^{1}$. Par ailleurs, le titre de l'article de Kurylowicz, traduit de l'anglais en français pour Diogène, se trouve être aussi le titre, à un synonyme près, d'un article de Benveniste paru en 1966, traduit du français en anglais pour Directions for Historical Linguistics et repris dans PLG 2, sur "la transformation des catégories grammaticales", article où il est également beaucoup question du parfait et du futur ${ }^{2}$. La comparaison semble donc s'imposer entre ces deux recherches contemporaines sur un même problème, d'autant que leur réception, en dépit d'une égale fécondité, n'a pas été vraiment comparable: il n'est que de comparer le nombre de références respectives dans les textes théoriques de linguistique générale en français. Je voudrais montrer ici 1) la convergence de ces deux recherches, 2) à travers leurs points de divergence, leur différente fécondité heuristique, 3) l'actualité, toujours, de la comparaison de l'évolution historique dans la théorie générale, ce que Kurylowicz appelait modestement ses "recherches étymologiques".

Commençons par jeter un coup d'oeil sur les articles jumeaux (ceux de Diogène en 1965) que j'ai mentionnés en commençant :

La catégorie $d u$ passé se nourrit perpétuellement de formes qui, étymologiquement, sont des formes $d u$ présent, c'est-à-dire se rapportent au

\footnotetext{
1 Benveniste: "le langage et l'expérience humaine" (pp. 3-13), repris dans PLG 2, pp. 67-78.. Kurylowicz: "L'évolution des catégories grammaticales (pp. 54-71). Entre les deux, Chomsky, Jakobson et Martinet, dans Diogène 51.

2 "La Transformation des catégories grammaticales", PLG 2 (pp. 126-136). Originalement contribution au Symposium for historical linguistics, 29-30 avril 1966. Publié en 1968 dans Direction for Historical Linguistics, Univ of Texas Press, Austin London pp. 85-94.
} 
Annie Montaut

moment où l'on parle. Le changement sémantique n'est possible que parce que la forme originale indique un état présent (résultant d'une action passée) et non pas une action présente" (l'auteur souligne, p. 5)

"L'origine du futur offre avec le passé des analogies frappantes (will, shall, am going to, fr-ai, etc...). Toutes ces tournures sont à l'origine des formes $d u$ présent, c'est-à-dire qu'elles se rapportent au moment où l'on parle. Elles n'expriment pas l'action mais l'obligation, le désir, l'intention, d'agir. Tout comme le résultat présent d'une action antérieure sous-entend que l'action a eu lieu avant le moment où l'on parle, de même le désir présent d'agir suggère que l'action elle-même aura lieu après le moment où l'on parle, c'està-dire dans le futur" (K. p. 59)

$C^{\prime}$ est donc à la situation du dialogue, et à la notion fondamentale d'en ce moment (now), ce moment où l'on parle, qu'il faut toujours en revenir, non seulement pour établir le jeu des relations temporelles [...] mais aussi pour créer de nouvelles formes d'expression verbale du passé et de l'avenir, qui, en raison même de leur lien encore perceptible avec la situation concrète du dialogue, sont plus expressives que les anciennes" (K. p. 60)

Le seul temps inhérent à la langue est le présent axial du discours, (...) Il détermine deux autres références temporelles; celles-ci sont nécessairement explicitées dans un signifiant et en retour font apparaître le présent comme une ligne de séparation entre ce qui n'est plus présent et ce qui va l'être. Ces deux références ne se reportent pas au temps, mais à des vues sur le temps, projetées en arrière et en avant à partir du point présent. (B. p. 9).

Nous noterons un fait significatif. On constate que dans les langues des types les plus variés, la forme du passé ne manque jamais, et que très souvent elle est double ou même triple. (...). Au contraire, beaucoup de langues n'ont pas de forme spécifique pour le futur. (...)

Ce contraste entre les formes du passé et celles $d u$ futur est instructif par sa généralité même dans le monde des langues. Il y a évidemment une différence de nature entre cette temporalité rétrospective, qui peut prendre plusieurs distances dans le passé de notre expérience, et la temporalité prospective qui n'entre pas dans le champ de notre expérience et qui à vrai dire ne se temporalise qu'en tant que prévision d'expérience. La langue met ici en relief une dissymétrie qui est dans la nature inégale de l'expérience (B. p. 10)

Les points communs sont évidents. Le rattachement des deux références passé et futur au présent, érigé en point axial et générateur, est central dans les deux analyses. C'est Benveniste qui emploie les deux adjectifs: "ce temps [linguistique] a son centre - un centre générateur et axial ensemble - dans le présent de l'instance de parole" (p. 8). Toutefois, si la notion d'axialité est longuement argumentée chez Benveniste, c'est chez Kurylowicz qu'on trouve des commentaires et des exemples de formes aussi précis qu'abondants sur 
l'aspect générateur du présent dans l'expression du temps linguistique. A l'appui de la première citation (la catégorie du passé se nourrit perpétuellement de formes du présent) Kurylowicz cite l'anglais, l'allemand, le français, (am, have, habe, ai), et l'indo-iranien, référence familière aussi de Benveniste, et détaillée dans un autre article de Kurylowicz sur "Aspect et temps dans l'histoire du persan" (1953). J'en donne un exemple, car il permet de voir nettement comment Kurylowicz voit la génération des formes d'expression du temps dans la langue. Décrivant l'évolution diachronique du système iranien, il montre que la forme kardam, aujourd'hui "fis", (< kartam, adjectif verbal ou ppp avestique "fait"), avant de se substituer aux formes de passé narratif et d'aoriste, a eu une valeur mixte, ce qu'il appelle l'"aspect complexe" à la fois perfectif et imperfectif ${ }^{3}$. Une fois établie la substitution de la forme à l'ancien aoriste et à l'ancien parfait, se crée une nouvelle forme pour prendre en charge l'aspect complexe: kardah am (participe + copule) ${ }^{4}$. Ce renouvellement des formes est en quelque sorte modélisé par les fameux losanges de Kurylowicz (deux termes, positif et négatif, et deux termes résultant de leur produit, complexe et neutre): un système d'aspects complet comporte en effet les quatre termes :

GAMA neutre

(ni imperfectif ni perfectif : présent dit général)

BETA négatif : imperfectif (présent duratif)

$$
\begin{gathered}
\text { gama complexe } \\
\text { (formes exprimant l'état, "il est maigri") }
\end{gathered}
$$
beta, positif : perfectif (parfait latin scripsi)

De même qu'on observe une affinité entre beta et gamma, conduisant au fréquent renouvellement de beta par gamma (scripsi renouvelé par scriptum habeo < scriptum mihi est, yaktul perfectif de tuer par katal étymologiquement nom d'agent en sémitique occidental), de même l'affinité de GAMA neutre et BETA imperfectif (négatif) conduit au renouvellement de GAMA par BETA (par exemple du présent dit général par le duratif comme pripekajo, ancien itératif, qui a complètement supplanté l'ancien présent pripeko). Ainsi le renouvellement des formes linguistiques peut se représenter par une rotation du losange, dans le sens des aiguilles d'une montre.

Quant au système temporel, il correspond au dédoublement du système aspectuel, et Kurylowicz propose de considérer la divergence entre langues

\footnotetext{
${ }^{3}$ Les termes de perfectif et imperfectif, qui sont de K., peuvent, ici, être "traduits" par accompli et non accompli. On se gardera toutefois de présenter ces deux paires comme des synonymes, notamment dans la description de langues qui ont grammaticalisé les deux oppositions (bulgare par exemple).

${ }^{4}$ Pp. 111-14. . Carey (1994) montre également (tout en s'opposant à Kurylowicz en ce qui concerne la nature de l'objet des prédicats qui peuvent entrer dans cette évolution) que la valeur stative (adjectivale) est bien constitutive des parfaits en "have" du vieil anglais. Ce n'est qu'en moyen anglais qu'on trouve des marques de la valeur processuelle ("perfective") de la structure.
} 


\section{Annie Montaut}

dites à aspect et à temps comme "une différence d'ordre quantitatif, reposant sur un degré plus ou moins avancé de grammaticalisation de certains traits sémantiques du verbe". Autant dire qu'il relève aussi du renouvellement des formes.

Revenons au commentaire des analogies et des différences de notre premier couple de citations. Le futur, dont Benveniste ne parle guère ici que pour souligner sa dissymétrie avec le passé, est analysé chez Kurylowicz dans l'histoire de ses représentations linguistiques comme une continuelle régénération par l'emploi de formes de présent, ou perfecto-présent. C'est une analyse très détaillée des formes, et encore une fois de l'histoire de ces formes exprimant les deux références au passé et au futur qui permet à Kurylowicz de conclure, comme d'ailleurs Benveniste qui pose cela d'emblée, que ces deux types de références se caractérisent par leur rattachement au moment de la parole, et non par la représentation d'actions passées ou futures. Par contre, si tous deux aboutissent au caractère axial de la situation de dialogue, Benveniste choisit de donner à cette notion non seulement une position centrale (comme Kurylowicz le fait aussi dans son analyse des cas et de la représentation de l'espace, des pluriels) mais un rôle d'ouverture sur la notion d'intersubjectivité. Celle-ci permet de convertir en "omnipersonnel" un temps à vocation unipersonnelle (p. 11) .Benveniste fait donc de l'analyse du temps un tremplin pour une théorie de l'énonciation. Alors que Kurylowicz, après avoir analysé d'autres catégories linguistiques caractérisées également par leur ancrage sur la situation de dialogue, appuie tout au long son argumentation sur le renouvellement des formes, et procède des catégories grammaticales aux "superstructures", qui, trouvant leur justification à un niveau plus abstrait, s'appuient sur des catégories fondamentales du système linguistique et mettent en évidence les fonctions essentielles du langage : représentation ou symbolisation (passif par rapport à actif), mais aussi expression, ou invocation (origine du subjonctif, de l'optatif, de l'impératif), liant explicitement, par l'enquête sur la convertibilité des catégories, les "recherches étymologiques" à l'interrogation théorique sur le langage.

Avant de revenir en conclusion sur ces différences de perspective, continuons à observer le détail de ces articles jumeaux. Outre le poids divergent accordé à la notion d'axialité et de générativité du présent, que je viens de commenter, il y a une autre dissonance ; aussitôt posée l'analogie du passé et du futur dans leur lien au présent de l'énonciation, Benveniste les distingue nettement et conclut à une dissymétrie profonde et "naturelle", car relevant de l'expérience humaine la plus générale. Ces deux références, bien qu'étant identiquement des "vues depuis le présent", ne sont donc nullement superposables. Chez Kurylowicz en revanche, c'est la symétrie qui est soulignée, et la symétrie de l'histoire des formes, que Benveniste n'aborde pas dans l'article de 1965. Différences ténues, estimera-t-on. Mais si on regarde d'autres articles consacrés à la question par chacun des deux auteurs, les différences auraient tendance à s'accuser en opposition. C'est du moins comme 
cela que les formule Benveniste, prenant explicitement parti contre une théorie, erronée, fauteuse de confusion, pour en proposer une autre, la sienne, radicalement distincte. Dans l'article cousin cette fois (sur les catégories grammaticales) par exemple, où les deux questions du parfait et du futur sont abordées de façon synthétique, Benveniste pose des dichotomies tranchées entre thèses adversaires, l'une, bien connue, entre origine passive et possessive $\mathrm{du}$ parfait transitif, l'autre un peu moins, entre origine prescriptive et "prédestinative" du futur. Tranchant de la dichotomie : "ce qui est erroné au triple point de vue de la description, de l'histoire et de la théorie générale, et qui installant la confusion au coeur du problème, empêche même de le poser" (pp. 130-1) $)^{5}$.

Il faut donc en penser que Kurylowicz, qui n'est pas cité malgré l'abondance des références sur la thèse condamnée, et malgré l'importance d'au moins deux de ses articles sur la question (1931, 1953), repris dans Esquisses Linguistiques, s'oppose directement à Benveniste qui lui donne implicitement le rôle de tenant de la doxa erronée. De fait, il ne met à aucun moment en question la thèse de l'origine passive du parfait, il apporte au contraire à Schuchart, qui avait en quelque sorte lancé cette thèse (après Geiger), un élément important de justification. Et il interprète l'histoire du futur périphrastique dans un strict parallèle avec celle du parfait périphrastique, sur des données indo-européennes qui étaient aussi celles de Benveniste (vieux perse, sanscrit, latin, langues romanes). Voici les données, bien connues

tya manâ krtam, tat mama/mayâ krtam, id mihi factum est, habeo factum, j'ai fait avestique sanscrit latin

La différence de position tient à ceci que la structure, interprétée depuis comme passive (ceci par moi est fait > j'ai fait ceci), Benveniste l'interprète comme possessive, à cause de la différence casuelle, sur laquelle il insiste beaucoup. L'agent est au génitif (vx perse) ou au datif (latin), cas ordinairement utilisés pour exprimer la possession avec un prédicat existenciel (mihi est filius), et radicalement distinct de l'agent du passif, ablatif prépositionnel (hacâma vx perse) ou instrumental (mayâa sanscrit). "Cette différence dans la forme casuelle du pronom manâ d'une part, hacâma de l'autre, montre que le parfait doit s'interpréter comme une catégorie propre, et qu'il est en tout cas distinct du passif (...) c'est un parfait actif d'expression possessive" PLG1, 179-80).

Cette différence casuelle n'échappe pas à Kurylowicz, mais il ne lui accorde pas une importance centrale: "le tour mihi factum est, expression

5 ..."ni les une ni les autres [conditions précises du tour latin, précisions théoriques] ne semblent encore pleinement reconnues" (...) de là l'état de confusion qui règne au sujet de cette question" (129). A propos du futur, même ton péremptoire: (131-2). Encore : "il faut bien dire que cette manière de symboliser le passage d'un état à l'autre est erronée, à la fois dans la réalité historique, si elle prétend à la résumer, et comme modèle théorique, si elle prétend à la faire comprendre (...) Cette double erreur, historique et théorique, résulte elle-même d'une interprétation inexacte du syntagme habere+ infinitif" (PLG2, 131) 
Annie Montaut

idiomatique attestée dès l'époque de Plaute, n'est en réalité qu'une construction passive. Car mihi factum est, avec son datif dit dativus auctoris, équivaut à a me factum est" 6 .

Je me garderai de prétendre départager deux autorités sur l'équivalence ou la non équivalence des deux cas, mais me bornerai à signaler ici que, si l'évolution du persan montre bien la prééminence du génitif dans le tour vieux perse qui précède le parfait actif du persan moderne, par le fait que le genitif vx perse manâ s'est recatégorisé comme pronom sujet (nominatif) man "je" en persan moderne, c'est au contraire l'instrumental mayâ et non le génitif mama en sanscrit (selon Benveniste concurrentiels, mais il ne retient pour son argumentation que mama), qui s'est recatégorisé comme pronom sujet (nominatif) de permière personne en hindi moderne.

Si Kurylowicz passe légèrement sur l'importance de la distinction casuelle, la distinction temporelle, ou aspectuelle, par contre, lui est essentielle. C'est elle qui lui permet, par ailleurs, de justifier, dans une même argumentation, la formation des parfaits et des futurs périphrastiques en avoir dans les langues romanes. Tous deux en effet sont analysés par Kurylowicz en opposition avec le présent :

\begin{tabular}{|c|c|c|c|}
\hline $\begin{array}{l}\text { mihi occisum est } \\
\text { habeo occisum } \\
\text { j'ai tué -actif } \\
\text { vues depuis (en al }\end{array}$ & $\begin{array}{l}\text { mihi occidendum est } \\
\text { habeo occidendum } \\
\text { je tuer-ai -actif } \\
\text { /en avant) le présent }\end{array}$ & VS & $\begin{array}{l}\text { a me occidetur } \\
\text { qui ne se convertit } \\
\text { jamais en valeur active } \\
\text { action présente }\end{array}$ \\
\hline
\end{tabular}

Selon Kurylowicz le tour passif se convertit en parfait transitif actif, selon un processus de causativation; de même certains parfaits d'intransitifs ont pris avec avoir en ancien roman une valeur causative : mourir, courir, par exemple, ont au parfait auxilié avec avoir le sens causatif de tuer, chasser, totalement absent du latin, et impossible au présent, où "je meurs" ne peut jamais signifier "je tue" alors que "j'ai mort" signifie "j'ai tué"7. D'où la reconstruction de la structure en partant, à l'origine, de verbes ayant un objet externe nettement distinct. L'emploi d'“avoir, have" en anglais se serait seulement ensuite généralisé à des verbes abstraits comme "comprendre, connaître", et même, ultérieurement en anglais aux intransitifs (he has gone, vs he is gone, qui est statique et non parfait). A l'inverse, Benveniste reconstitue la structure en l'associant, d'abord, à des verbes de perception, à diathèse quasi interne, comme "comprendre", puis seulement par extension secondaire à des

\footnotetext{
6 "Temps composés en persan" repris dans Esquisses Linguistiques, p. 106

7 L'exemple de l'anglais (to have a book written, vs to have written a book) est aussi donné comme argument en faveur d'une origine causative (avoir causativant le passif) du parfait: avant que l'ordre des constituants n'ait fixé ces deux tours dans des sens distincts, ils auraient, à une époque donnée, été des quasi variantes, l'un insistant plus sur l'état, l'autre sur l'action à l'origine de l'état. Diogène, p. 57. Carey (1994) suit dans l'ensemble Kurylowicz, montrant qu'en vieil anglais la valeur stative est constitutive des parfaits en "have", la valeur processuelle n'apparaissant qu'en moyen anglais.
} 
verbes comme "écrire, tuer", etc. L'idée de conversion causative (perception linguistique de la structure comme active) de constructions passives exprimant parallèlement le passé et le futur rend compte de la symétrie entre ces deux "temps". A la source du futur Kurylowicz donne la construction parallèle à mihi factum est, mihi cantandum est, fréquent à l'époque post-classique, et généralisé pour exprimer le futur passif : torquendus es quia homicidia es (tu seras torturé car tu es homicide $=$ on te torturera). Sans doute, l'étape médiane qu'il mentionne, comme tous les "manuels" qu'accable Benveniste, cantare habeo, est à corriger en cantari habeo. Il n'en reste pas moins que la structure à l'origine du futur périphrastique en habere, est datif + adj verbal passif (gérondif) + esse, c'est-à-dire le même type de construction qui est à l'origine du parfait. D'où le parallélisme :

Dans l'évolution passif > actif qu'on vient d'étudier, le pas décisif consiste dans le remplacement de datif + esse + nominatif par nominatif + habere + accusatif. La construction passive a été transformée en une construction active $^{8}$.

Un tel parallélisme ne pouvait être mis en évidence par Benveniste, car, pour commencer, le parfait n'a selon lui pas une origine passive mais possessive. Quand il s'intéresse au futur, prenant le contrepied de la doxa dans une brillante démonstration, massivement illustrée, il ne mentionne pas le tour d'origine, c'est-à-dire la substitution généralisée d'un premier tour périphrastique (datif-gérondif-esse) au tour synthétique. Il part directement des premières attestations du tour avec habere, auquel il dénie toute association avec une quelconque idée d'obligation ou d'envisagement futur :

habere ne signifie pas "avoir (à)", comme dans "j'ai à travailler". Sens qui n'aurait jamais conduit à un futur "je travaillerai" et qui en est même si différent qu'aujourd'hui comme autrefois " $j$ 'ai à travailler" ne correspond jamais à "je travaillerai". Dans le syntagme latin tel qu'il s'est constitué, [en subordonnée au début, uniquement à l'imparfait d'habere, et avec l'infinitif passifl, habere avec l'infinitif a pour fonction d'indiquer la prédestination de l'objet désigné à être fait tel (...) indiquant non l'obligation (comme le fait la forme en -ndus), mais la prédestination.

Il ne s'agit pas ici d'aller vérifier l'exactitude des affirmations (antériorité des premières attestations chez Benveniste, ou à l'inverse l'extension de la substitution du tour périphrastique en -ndus au futur passif synthétique chez

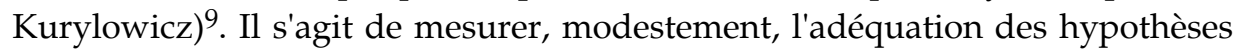
respectives à des données empiriques différentes, de manière à pondérer la pertinence de telle ou telle assertion en ce qui concerne la théorie générale,

\footnotetext{
8 "Les Temps composés du roman" (1931), in Esquisses p. 107.

9 Ni de départager les tenants de ces théories opposées, qu'on retrouve de Vogt (1950) à Allen (1964), qui mettent en évidence grâce à ces hypothèses des faits linguistiques intéressants, concernant l'ergativité et la transitivité.
} 


\section{Annie Montaut}

puisqu'aussi bien, les tenants des thèses classiques (parfait passif, futur d'obligation) sont nettement accusés par Benveniste de semer la confusion, de fausser les termes du problème au point d'empêcher même de le poser, bref, d'être gravement erronés sur le triple plan descriptif, historique et théorique.

Les données indo-aryennes me serviront d'exemple : elles sont à la fois parallèles à celles de l'iranien (extrapolées à l'"indo-iranien" dans les deux cas) largement utilisées par les deux auteurs, et divergentes par l'évolution des vernaculaires modernes et contemporains (NIA ancien et moderne). Le parfait transitif ${ }^{10} \mathrm{du}$ hindi moderne standard, représentatif du groupe nord occidental de l'IA contemporain ${ }^{11}$, offre la particularité d'être ergatif: à l'accompli, le "verbe" s'accorde avec l'"objet", et l'agent est marqué par l'oblique suivi d'une postposition spécifique (ne ). En quoi il se distingue radicalement, et du parfait intransitif (1b) et de l'inaccompli (1c) :

$$
\begin{aligned}
& \text { bacce ne kahânî paRhî } \quad 1 b \text { aur (vah) sone gayâ } \\
& \text { enfant-ms-obl ERG histoire-fs lire-fs et (il) dormir aller-ms }
\end{aligned}
$$

l'enfant lut une histoire et (il) alla se coucher

$1 \mathrm{c}$

$$
\text { bacca : kahânî paRh rahâ hai l'enfant est en train de lire une histoire }
$$$$
\text { enfant-ms-nom histoire-fs lire progr-ms être-3s }
$$

Cette construction, dite ergative, est historiquement issue de la généralisation des structures dites passives dès le sanscrit classique: mayâ : /mama tat krtam (moi-instr ceci-ns fait-ns) est l'expression régulière du procès passé transitif, qui remplace le riche paradigme conjugué du védique. Voir en 2a, l'"ancêtre" (Asroka, troisième siècle avant JC, prakrit occidental) de la construction ergative du hindi : pour dire que le roi Asoka a fait graver l'édit de la loi, on a 'l'édit (est, a été) gravé par le roi Asoka' :

2 a) iyam dhammalipî devânampriyena priyadassina râññâ lekhâpitâ. cetteloiécrit-fs dieux-gen-chéri-instr au-regard-amical-instr roi-instr inscrit-caus-fs "le roi ami des dieux au regard amical a fait écrire cette loi"

2b) iyam dhammalipî devânampiyena piyadassinâ [lâjinâ] lekhitâ. ${ }^{12}$

L'énoncé est en fait une prédication nominale (en fonction prédicative, un adjectif verbal de sens passif) comportant un terme au nominatif, celui sur lequel se porte le résultat du procès, et un terme instrumental représentant l'agent source du procès. En hindi moderne, si l'énoncé est aujourd'hui perçu comme processuel et "actif", l'agent étant d'ailleurs en première position dans la séquence ${ }^{13}$, il reste formellement non verbal, ne présentant que des

10 Les termes parfait, transitif, objet, sujet, ne sont bien sûr pas sans poser problème, dans les énoncés en question, à acteur au datif. Je leur conserve l'acception que leur confère Benveniste

11 Toutes dépourvues d'équivalent lexical du verbe avoir, donc n'ayant pu accomplir la transformation active de la structure passive d'origine par l'auxiliation en avoir, à la différence de certains dialectes du persan (sogdien, chorasmien, persan oriental, ont des parfaits en dâr, "avoir").

12 Les Inscriptions d'Asoka, Belles Lettres

13 Dans les énoncés non marqués. 
désinences de type adjectival (ni temps, ni personne, ni même diathèse), et exhibant toujours de claires affinités avec le statique --en quoi elle s'oppose clairement au passif, processuel et dynamique, récemment créé ${ }^{14}$.

Dans le système relativement bien conservé du hindi, il apparaît peu pertinent d'opposer origine possessive et origine passive (beaucoup plus vraisemblable du fait des marques casuelles d'origine), le syncrétisme casuel dat-gen-instr : oblique ait dès le $10-11^{\circ}$ siècle effacé les choses ${ }^{15}$. Ce qui est certain, c'est qu'on a affaire dès le moyen indien, à une prédication nominale : l'expression d'un processus transitif passé a tôt consisté à nominaliser le résultat du procès (d'où ses affinités encore perceptibles avec le statif), et à attribuer secondairement ce résultat à une source marquée, non au datif $(k o)$ mais à l'ergatif, qui semble bien issu de l'ancien instrumental (maiN 'je" < mayâ, une fois perçu comme sujet actif, est recaractérisé comme agent oblique par ne).

On peut se demander pourquoi les langues IA orientales comme le bengali, héritières de $2 b$, prakrit magadhéen de même structure que les prakrits occidentaux $2 \mathrm{a}$, ont un parfait transitif franchement actif, analogue au parfait intransitif, et comme lui pourvu de désinences personnelles, apparemment d'un affixe temporel, et d'un sujet nominatif ${ }^{16}$ :

ami poR-il-am, tui poR-il-e, tumi poR-il-e je lus, tu lus, vous lûtes je-nom lire-"passé"-1ํ, tu-fam lire-"passé"-2, tu-hon lire-passé-2-hon

C'est que la perception de la structure comme active a entraîné la réfection du système (l'activation", dit Chatterji), comme en persan moderne (man kardam, to kardi, u karde, etc). En réalité, historiquement, ce qui est

14 Comme l'indique la possibilité d'adjoindre le statif hua : dans le paradigme de 1a à orientation présente (passé composé) :

is mahilâ ne aTahru pahne hue haiN

cette femme-obl erg pendants-d'oreille-mp porter-mp hua-mp être-prst-mp

"cette femme a mis (porte) des pendants d'oreille"

Cet énoncé entre certes dans un paradigme (à trois formes) où des marqueurs de temps (présent ici) ont pu réactiver la valeur verbale et où donc se trouve effacée la nature proprement nominale de la forme de base, conférant du même coup à la forme de base, à l'origine adjectivale, une valeur perçue comme processuelle. Mais ce sont des créations récentes, et la forme de base, anciennement dite "non définie", c.à.d. non marquée pour le temps, comportait intrinsèquement le trait statif que hua: aujourd'hui peut ajouter aux deux autres formes du paradigme. Pour plus de détail sur les structures ergatives modernes, voir A. Montaut 1993.

15 Certes les grammaires traditionnelles décrivent la structure comme "karmani" , passif --"bhave " ou moyen lorsque l'objet, marqué $+k o$, ne contrôle plus l'accord verbal : unhõ-ne laRkî : ko dekh-â : (ils-obl-erg fille-fs acc regarder-pft-n/ms), "ils virent la fille". Mais il faut faire la part des calques notionnels ( $\mathrm{du}$ sanscrit en l'occurrence), et l'existence, nettement distincte d'un passif périphrastique en hindi moderne (flexion verbale et marque de l'agent distincts de l'ergatif) montre l'inadéquation de l'analyse passive, en synchronie. Quant à l'analyse active (grammaires modernes), qui relègue au rang des reliques historiques sans pertinence profonde la marque ne et les marques casuelles, elle pose évidemment de graves difficultés, puisqu'elle identifie construction nominative de l'inaccompli et construction ergative de l'accompli.

16 Prakrit magadhéen : de la région de Magadha, relativement orientale. 


\section{Annie Montaut}

aujourd'hui perçu comme un morphème de passé (il, l), est l'ancien suffixe d'élargissement des bases nominales en -ila, -illa ${ }^{17}$. Si bien que le système perfectal du bengali ne garde pratiquement plus de traces de l'ancienne structure. Mais jusqu'au $\mathrm{XV}^{\circ}$ siècle, date où le nouveau système "réactivé" est vraiment établi dans la langue, on trouve des constructions à agent oblique (4a), et, même anciennement, avant que ne soit complètement estompée la distinction des genres, des structures où l'adjectif verbal s'accorde avec le patient $(4 b)$ :

4a ebeN maï bujhila (caryâ 35, glosé "by me it has been understood" par Chatterji)

maintenant je-instr comprendre-il-n, "maintenant j'ai compris"

4b Kona purâne, kânha, hena sun-il-î kâhin-1̂,

quel purana-loc, Krishna, ainsi entendre-il-fs histoire-fs

"dans quel purana, o K, cette histoire a-t-elle été racontée, a-t-on raconté cette histoire"

Le marathi (sud occidental) offre un état encore différent de cette "activation" de l'ancienne structure passive : adjonction (et non substitution) de désinences personnelles et passage de l'agent au nominatif à la $2^{\circ}$ et $3^{\circ}$ personne, mais maintien d'un morphème d'accord avec l'objet.

Quant aux futurs, c'est dans les langues IA orientales que peut se vérifier l'analogie avec le parfait (le hindi ayant adopté un futur périphrastique sur la base de l'ancien présent auxilié avec un participe du verbe "aller"). Elle est nette dans la synchronie du bengali moderne, où le système de désinences du futur est le même que celui du parfait $\left(-\mathrm{o},-\mathrm{e},-\mathrm{i}\right.$, le $-\mathrm{o}$ de $1^{\circ}$ personne représente une sorte d'allomorphe de la consonne labiale selon Chatterji), et distinct de celui du présent (ami poR-i, tui poR-is, tumi poR-o). Elle est surtout nette dans l'histoire de la structure, qui a subi exactement la même "activation" que celle du parfait, et dont on trouve quelques rares témoignages en vieux bengali :

5 mai dibi piricha <scrt: mayâ datavyâ pricchâ

je-instr donner-part-passif-fut-f questions

"je poserai une question"18

Les futurs orientaux en -b-, où -b- est couramment analysé, parallèlement au passé, comme morphème temporel de futur, sont en effet hérités, non du futur sigmatique sanscrit (ex désidératif), mais de l'adjectif

17 Qui semble avoir été utilisé comme l'élargissement -aka des dialectes occidentaux, et qu'on retrouve encore dans des termes comme aglâ, "prochain", pichlâ, "précédant, dernier", kânTilâ, "épineux", rangilâ, "coloré", tous des nominaux. Cet "élargissement" en -l- se retrouve dans les passés modernes de dialectes orientaux du hindi (awadhi, bhojpuri), comme en bengali, mais aussi en marathi.

18 Exemples empruntés à Chatterji pp. 808 et 967, Caryâ 35, 5 et 29 respectivement. L'auteur signale dans le chapitre des pronoms que mae, maï, est un vieil instrumental ("as yet instrumental in $\left.\mathrm{OB}^{\prime \prime), " u s e d ~ i n ~ t h e ~ p a s s i v e ~ c o n s t r u c t i o n ~ w i t h ~ t h e ~ p a s t ~ o r ~ f u t u r e ~(i n ~ "-i l, ~}-i b-"\right)$. 
verbal passif à valeur d'obligation en -tavya $(=-n d u s)$. Ce n'est que vers le $15^{\circ}$ siècle que se substituent les désinences personnelles aux marques d'accord nominales (Genre Nombre), en même temps que les groupes obliques désignant l'agent se convertissent au nominatif. Les dialectes orientaux du hindi fournissent des données analogues ${ }^{19}$; quant au marathi, qui maintient la valeur modale de la forme héritée (en -av, -âv <-tavya), il maintient aussi des constructions à agent oblique : le potentiel et l'obligation reflètent la situation du parfait (avec la possibilité d'une reconversion "active" dans le cas de l'obligation)

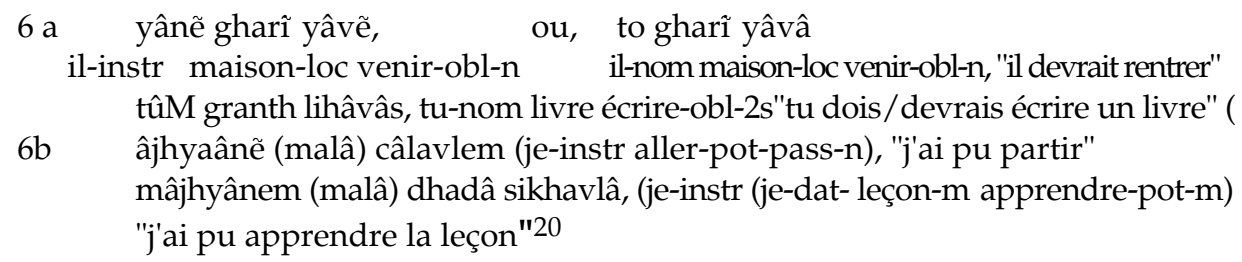

Les données IA établissent donc que le parfait transitif ne semble pas avoir eu un "sens possessif", mais représenter une transformation active de la structure passive à agent instrumental, transformation nettement marquée par le renouvellement morphologique en bengali, parler plus novateur. Quant aux futurs en $-b-$, ils ont nettement leur origine dans l'adjectif verbal passif à valeur d'obligation, et n'ont à aucun moment marqué la prédestination. Leur histoire en bengali est rigoureusement parallèle à celle du futur. Il paraît donc hâtif d'imputer aux théories du parfait "passif" et du futur d'obligation, une cécité historique et théorique. Il ne fait pas de doute que Benveniste procède largement par contre pied. Contrepied des positions admises, des évidences, des dernières découvertes. On peut dire sans offenser sa mémoire qu'il a l'esprit de contradiction, l'esprit et souvent le ton, péremptoire. On peut aussi appeler ça le goût du paradoxe. Mais si le goût du paradoxe a ses côtés irritants, il mène parfois à des trouvailles illuminantes. Si j'ai pu avoir l'air de "donner raison" par les données IA à Kurylowicz, lequel capture une analogie, historiquement et théoriquement d'une grande portée me semble-t-il, il faut dire aussi que Benveniste, grâce à sa vision "possessive" du parfait, en capture une autre, non moins capitale pour la théorie générale. C'est le retournement que représente "avoir" par rapport à "être à" (mihi filium est, mihi factum est), l'analyse d'avoir comme inversion d'être à, comme verbe statif participant à des prédications de localisation.

Le terme même (prédication de localisation) n'est pas de Benveniste, mais ceux d'inversion, de retournement, d'extériorisation du sujet par rapport

\footnotetext{
19 Il en va de même en awadhi dialecte oriental du hindi. Saxena sinale au chapitre des pronoms que mai, ancien instrumental, est utilisé de préférence au nominatif hu, < scr aham, avec les participes passés et futurs (ie : formes de parfait, et futur en -b)

20 Exemples empruntés à Bloch, pp. 264-5.
} 


\section{Annie Montaut}

à la prédication, le sont (PLG1, pp. 197, 199) ${ }^{21}$. On peut représenter la différence entre inaccompli (sujet nominatif, intrinsèque à la prédication) et accompli ("sujet" extrinsèque) en termes de spatialisation. L'abondante littérature contemporaine sur l'aspect recourt le plus souvent à ce type de métaphore pour distinguer entre procès inaccompli et procès accompli (Fuchs 1978, Cohen 1989, Guentcheva 1991). Le premier type de procès, inaccompli, est ordinairement représenté comme un espace dont les limites ne sont pas prises en compte, qu'il soit borné d'un seul côté ("j'écris cet article"), ou des deux ("j'écrivais cet article") - l'ouvert, ou plutôt l'espace dont les bornes sont infiniment distantes et donc non perceptibles et non représentables, correspond à l'état. Le sujet de "écris, écrivais", dans "j'écris en ce moment un article, j'écrivais cet article quand le téléphone m'interrompit" est situé dans les bornes qui peuvent métaphoriser les limites initiale (à gauche) et finale (à droite) de ce procès. Il est donc localisé à l'intérieur du procès et par le procès. Des formulations classiques comme 'procès pris de l'intérieur, dans son développement' disent aussi, avec "intérieur", la même représentation spatialisée qui traduit, de façon plus ou moins constante, qu'il y a un intérieur et un extérieur au procès d'action ${ }^{22}$. $\mathrm{Si}$, donc, le procès non accompli est représentable comme localisant son sujet, directement impliqué, à l'intérieur de ses limites, il n'en va pas de même dans le procès accompli. Dire "j'ai écrit cet article" suppose que je suis sorti du procès, que je l'ai traversé de part en part pour me trouver, donc, à l'extérieur de la limite finale, à droite de la borne de droite, et c'est par rapport à la position extérieure que j'occupe alors, qu'est représenté le procès (article écrit) par le sujet, lequel n'est plus contenu dans les limites de l'action. Etant hors des bornes du procès, l'agent ne peut plus être localisé par le procès, il en est dissocié. Ce qu'on peut exprimer en disant qu'il n'est plus directement impliqué dans le procès. L'énoncé hindi de type 1a (mais aussi prakrit de type 2) matérialise la dissociation par la possibilité de ne même pas exprimer la source, le résultat pouvant s'énoncer comme tel, sans précision du lien avec sa source, sans localisation : ce qui donne un statique, ouvert ${ }^{23}$. Mais le procès prédiqué sur le terme d'arrivée (cible ou patient, ici "cet article"), peut aussi être localisé par rapport à 1 '“agent" désormais extérieur au procès, et formellement dissocié (cas oblique, souvent datif). Ce "dativus auctoris" du

\footnotetext{
21 "avoir n'est rien d'autre qu'un être-à inversé : mihi est pecunia se retourne en habeo pecuniam. Dans le rapport de possession indiqué par mihi est, c'est la chose possédée qui est posée comme sujet ; le possesseur n'est signalé que par ce cas marginal, le datif, qui le désigne comme celui en qui l'être-à se réalise. Quand la construction devient habeo pecuniam , ce rapport ne peut devenir "transitif" ; le "ego" posé maintenant comme sujet n'est pas pour autant l'agent d'un procès : il est le siège d'un état, dans une construction syntaxique qui imite seulement l'énoncé d'un procès" (197, "être et avoir dans leurs fonctions linguistiques")

22 Voir Culioli, 1971, 1990 (chapitre "Formes schématiques et domaines", pp. 115-126).

23 En hindi moderne : sâstrõ mẽ likhâ hai (traités dans écrire-pft-n), "c' est écrit dans les traités", Iit. (on) a écrit dans les traités. Seul le résultat est pris en compte. La forme est ergative (erg tronqué, ou court), mais le sens statique (espace ouvert)
} 
latin traduit le fait qu'avec l'accomplissement d'un procès transitif l'"agent" est extrinsèque, et n'est plus que le dépositaire (possesseur, attributaire) du résultat d'un procès à l'extérieur duquel il se situe ${ }^{24}$. Où l'on retrouve un type de structure locative c-à-d. une prédication localisée par rapport à un terme sans propriété locative (le possesseur, au cas oblique). C'est ce que M. Launey (1977) résume ainsi: "il faut bien voir que l'accomplissement d'un procès transitif donne naissance à un nouveau type de procès, imperfectif et statique, qui tend à être prédiqué sur le "terme d'arrivée", dans la mesure où, d'une part, le terme d'origine a été en quelque sorte expulsé du schéma transitif en acquérant des propriétés datives, et où, d'autre part, il y a de fortes chances pour que l'état en question soit l'état du terme d'arrivée", le plus souvent transformé par le processus" (p. 43). C'est ce que Kurylowicz (1953) montre pour le glissement de la forme kardam (<kartam) à des emplois de passé narratif, à partir d'une "valeur mixte", l'aspect complexe" à la fois "perfectif et imperfectif"25.

Pour revenir aux données indiennes, il est patent que le hindi exprime non seulement la possession, mais les prédications dont le sujet est siège d'un état ou d'un procès d'état, par des structures de type locatif: génitif de la possession inhérente (lien tout à partie), locatif de la possession contingente ("près de", relation d'adjacence), ou de la possession d'une qualité ("dans", relation d'inessence), datif de l'expérient, etc, avec le verbe être. Ram-ms gen deux soeur-fp (jambe-fp) être -3p un laRkõ mẽ himmat thî "ces garçons avaient du courage" ces garçons-mp dans courage-fs être-impft-fs

7c râm ke pâs bahut kitâbẽ haiñ "Ram a beaucoup de livres"

Ram près de beaucoup livre-fp être-fp

7d mujhko cintâ hai / bhûkh hai / usko angrezîâtî thî,j'ai du souci/faim /il savait l'anglais je-dat souci-fs est-3 / faim-fs est-3 /il-dat anglais-fs était-fs

Toutes ces structures offrent une analogie frappante avec le parfait transitif: marquage casuel oblique (+ postposition) de l'actant principal correspondant au sujet de avoir en français, accord avec l'autre terme, deux indices $\mathrm{du}$ caractère extrinsèque du premier par raport à la prédication. Benveniste note parfaitement ces analogies dans "être et avoir" (p. 198) : les prédicats statiques du Tunica de Louisiane désignant des états émotionnels, physiques, de possession, là où le français a avoir, ont une construction spécifique, impliquant des préfixes pronominaux (ceux-là mêmes de la possession inaliénable). Ces constructions en hindi présentent aussi avec le parfait une différence : c'est l'emploi obligatoire d'un verbe existenciel (distinct,

\footnotetext{
24 Dès lors qu'il est représenté comme dépositaire des résultats ou siège du procès, il est difficile de le considérer comme un agent véritable : d'où les guillemets.

25 voir supra. Les termes entre guillemets sont de Kurylowicz.
} 


\section{Annie Montaut}

dans les langues qui les distinguent comme le bengali, le népalais, le vieux marathi, de la copule), alors que le parfait simple (non marqué pour le temps, bien que par implication discursive il ait pris une valeur passée) est un prédicat nominal en hindi. Il serait trop long de creuser cette question, que Benveniste aborde dans "la phrase nominale" (et un peu dans "être et avoir", pp. 190-3), mais qu'il se garde de rappeler à propos de l'analogie parfait, être à, analogie qui passe par mihi factum est, après mayâ krtam, manâ kartam. Quoiqu'il en soit, l'analyse du parfait par Benveniste marque un tournant essentiel dans la description de l'ergativité scindée en fonction de l'aspect. Le caractère statif de telles prédications - que Kurylowicz voit aussi mais ne rattache pas aux autres types de prédications bi-actancielles statives - est mis ainsi en regard des autres prédications statives, ce qui est un point essentiel dans la théorie non seulement des auxiliaires avoir et être, mais de l'ergativité et de l'accompli.

Quant à l'analogie avec le futur (du moins d'origine modale : obligation), que fait Kurylowicz et non Benveniste, Launey dans l'article déjà cité la confirme par les données du nahuatl, (mêmes pluriels atypiques en - $k$, le même aussi que les prédicats issus d'adjectifs, que les noms d'agent). Si on reprend le petit schéma de spatialisation donné plus haut à propos de l'aspect imperfectif et accompli, on peut dire que l'agent d'un procès futur se situe, comme l'agent d'un procès accompli, à l'extérieur des limites du procès. Mais cette fois c'est à gauche de la borne gauche qu'il est situé, n'ayant pas encore franchi la limite initiale, et se représentant le procès comme un "être à pour lui", ou, de façon inverse, un "avoir à" (c'est à faire pour moi, j'ai à faire). C'est dire que ce n'est pas n'importe quel futur qui est susceptible de fonctionner comme un aspect non inaccompli. C'est le futur modal (à valeur d'obligation) et non temporel, ce dernier représentant probablement une édulcoration de la valeur initiale ${ }^{26}$. Dans les deux cas du parfait et du futur, et à l'inverse du procès inaccompli qui seul peut localiser son sujet à l'intérieur de ses limites, procès accompli (cible dépassée) et procès visé (cible à atteindre ou atteignable) sont localisés par rapport à un actant extérieur à la prédication.

Il est peu plausible que de telles analogies, présentes dans des langues aussi différentes que le nahuatl, l'IA, les langues romanes vues au prisme de Kurylowicz, relèvent de la simple coïncidence. Leur mise en évidence est d'une grande pertinence pour la théorie générale, ni plus ni moins que celle des analogies entre parfait et statiques. Le repérage de la prédication (statique, descriptive) par rapport au localisateur qu'est le "sujet", dissocié, constitue une même opération sous jacente à tous ces tours à actant oblique. La transformation active (avoir) efface, historiquement, les traces de cette opération, de sorte qu'on a affaire, au terme de la transformation à une opération différente (inversée, retournée, en ce qui concerne le repérage). L'évolution historique

\footnotetext{
26 S'il est fréquent de trouver des marqueurs de la modalité "vouloir" à l'origine du temps futur, il est rare de trouver "avoir" ou l'obligation (Bybee, 1991, Heine 1993 ne les mentionnent pas dans des ouvrages où sont pourtant passés en revue un grand nombre de langues).
} 
des structures en question montre que le renouvellement des formes, fonction de la perceptibilité de leur lien avec la situation de dialogue ${ }^{27}$, s'appuie sur la convertibilité des catégories syntaxiques. Où l'on retrouve la valeur heuristique de ce que Kurylowicz appelait ses "recherches étymologiques", pour la théorie générale. Si la comparaison des langues est aussi centrale à la théorie générale (Benveniste, Kurylowicz sont à leur façon des typologues), elle ne peut se faire à bon escient qu'en comprenant les structures modernes, interprétation qui passe souvent par le déchiffrement de leur histoire.

C'est le goût de histoire des formes et des structures qui conduit Kurylowicz à repérer l'analogie entre futur et parfait, analogie d'une grande portée théorique. Et c'est la contradiction des thèses passives sur le parfait qui conduit Benveniste à analyser les parfaits en avoir comme des constructions locatives, et à les associer aux prédications d'états subjectifs. Fidèle à sa méthode, fondamentalement structuraliste et volontiers polémique ${ }^{28}$, Benveniste débusque la pertinence de variations formelles trop vite jugées libres (datif et ablatif ou instrumental), et fait rendre raison sémantiquement aux différences formelles, justifiant le datif comme central à la structure "possessive", en même temps qu'il exige une base sémantique commune des constructions expérientielles et perfectales, formellement analogues. Mettant par ailleurs au centre de son analyse du temps le discours et la subjectivité - ce en quoi il déborde le structuralisme -, il interprète l'histoire du futur roman en l'axant sur les conditions discursives de son émergence, s'interdisant ainsi de capter l'analogie que Kurylowicz, parce qu'il part d'un autre point de vue, met en évidence. On peut vraiment dire que ces deux linguistes, également originaux, travaillant sur des problèmes et des données ici très proches, sont parvenus, par des moyens différents, à enrichir la théorie de l'aspect et du temps, et plus généralement de la prédication, sur deux points différents, mais également fondamentaux.

27 Comme le notait Kurylowicz dans son article de Diogène (p. 60) cité au début de cette étude.

${ }^{28}$ Sur la caractérisation de la méthode de Benvenites comparatiste, voir A. Montaut 1992. 
Annie Montaut

\section{Bibliographie}

ALLEN W.S., 1964, "Transitivity and possession", Language 40 (pp. 337-43)

BENVENISTE 1952, "La Construction passive du parfait transitif", BLS XLVIII, repris in Problèmes de linguistique générale 1, Gallimard (pp. 176-86)

BENVENISTE 1960, "Avoir et être dans leurs fonctions linguistiques", BSL LV, repris in PLG 1 (pp. 187-207)

BENVENISTE 1966, "Les transformations des catégories grammaticales", repris dans Problèmes de Linguistique générale, 2, Gallimard (pp. 127-136)

BLOCH Jules, 1914, La Formation de la langue marathe, nouvelle ed traduite et revue, 1970, Delhi, Motilal Banarsidass

Bybee, Pagliuca W. \& Perkins, 1991, "Back to Future", in Traugott E \& B. Heine, Approaches to Grammaticalization v.2, Amsterdam/Philadelphia, Benjamins (pp. 17-58)

CAREY K, 1994, "The Grammaticalization of the Perfect in Old English : an Account based on Pragmatics and Metaphors", in Pagliuca N. (ed) Perspectives on Grammaticalization , Benjamins, Current Issues in Linguistic Theory, 109 (pp. 103-116)

CHATTERJI, S.K., 1926, The Development of the Bengali Language, reed. 1986, 3 vol. Delhi, Rupa \&Co

COHEN David, 1989, L'Aspect verbal, PUF

Culioli A. 1971, "A propos d'opérations intervenant dans le traitement formel des langues naturelles", Mathématiques et Sciences Humaines, 34 (pp. 7-15)

Culioli A. 1990, Pour une linguistique de l'énonciation, Ophrys

DESCLÉE J.P., 1991, "La Prédication opérée par les langues (ou à propos de l'interaction entre langage et perception)", Langages 103 (pp. 83-96)

FuCHS C. 1978, "L'Aspect: un problème de linguistique générale", in Etudes sur l'aspect, Théorie et description, Paris, Publ. de l'Univ Paris VII, DRL (pp. 7-38)

GEIGER, 1893, "Die Passiv Construktion des Präteritum transitiver verba im Iranischen", Festgruss an Rudolf v. Roth

GUENTCHEVA Z, 1991, Temps et aspect : l'exemple du bulgare contemporain, ed. du CNRS

GURU KAMTAPRASAD, 1920, Hindi viakaran (Grammaire hindi) réed 1962, Benarès, Nagari Pracarini Sabha

HEINE B, 1993 Auxiliaries. Cognitive Forces and Grammaticalization, Oxford University Press

KELLOGG R.S., 1875, (réed 1972), A Grammar of the Hindi Language, Delhi, Oriental Book Reprints 
KURYLOWICZ J., 1931, "Les Temps composés du roman", réed. in Esquisses linguistiques, 1960, Wroclaw-Krakow, Polska Akademia Nauka (pp. 104-8)

KURYLOWICZ J., 1953 "Aspect et temps dans l'histoire du persan" (réed) in Esquisses linguistiques (pp. 109-118)

KURYLOWICZ J., 1965, "The Evolution of Grammatical Categories" Diogène 51 (pp. 51-71)

LAUNEY M. 1977, "Le pluriel transcatégoriel /-ke'/ en nahuatl ; contribution à l'étude de la relation "être" / "avoir"', Amerindia 2 (pp. 19-45)

MONTAUT A., 1992, "La méthode de Benveniste comparatiste : son discours et son sujet", LINX 26, Lectures d'Emile Benveniste, eds A. Montaut \& Cl. Normand (pp. 109-36)

MONTAUT A., 1993, "L'Interprétation de l'ergativité dans les langues indiennes", Modèles Linguistiques 28, 14-2 (pp. 89-104)

MONTAUT A. \& C. PilOt Raichoor, 1993, "Sémantique actancielle et cas morphologiques dans les langues indiennes", Langages 113 (pp. 90-114)

OJHA T, 1987, Pramukh bihari boliyõ ki tulna tmak adhyayan (Etude comparée des principaux parlers bihari), Vishvvidyalay Prakashan, Benarès

SAXENA, R.B., 1937, Evolution of Awadhi, reed 1971, Delhi, Motilal Banarsidass

SCHUCHARDT H., 1895 "Uber den passivem Charakter des Transitivs in den Kaukasischen Sprachen", SB Wien Akad. vol. 33

SHARMA A. 1958 (réed 1972) A Basic Grammar of Modern Hindi, Delhi, Ministry of Education and Social Welfare

TiWARI, U.N., 1966, The Development of Bhojpuri, Calcutta, The Asiatic Society (vol. X)

TIWARI U.N., 1961, Hindî bhâsâ kâ udgam aur uskâ vikâs , Prayag, Bharati Bhandar

VOGT J., 1950, "Un Aspect du problème actif passif dans le verbe", Journal de Psychologie $\left(\mathrm{n}^{\circ}\right.$ spécial Grammaire et Psychologie ) 